\title{
Analysis of Two Colliding Thin Spherical Shells
}

\author{
Yury Rossikhin, Vyacheslav Shamarin and Marina Shitikova \\ Research Center on Dynamics of Solids and Structures \\ Voronezh State University of Architecture and Civil Engineering \\ Voronezh 394006, Russian Federation \\ Email: yar@ vgasu.vrn.ru mvs@vgasu.vrn.ru
}

\begin{abstract}
In the present paper, the collision of two elastic spherical shells is investigated using the wave theory of impact. The model developed here suggests that after the moment of impact quasi-longitudinal and quasi-transverse shock waves are generated, which then propagate along the spherical shells. The solution behind the wave fronts is constructed with the help of the theory of discontinuities. Since the local bearing of the materials of the colliding elastic shells is taken into account, then the solution in the contact domain is found via the Hertz contact theory.
\end{abstract}

\section{INTRODUCTION}

The problems connected with the analysis of the shock interaction of thin bodies (rods, beams, plates, and shells) with other bodies have widespread application in various fields of science and technology. The physical phenomena involved in the impact event include structural responses, contact effects and wave propagation. These problems are topical not only from the point of view of fundamental research in applied mechanics, but also with respect to their applications. Because these problems belong to the problems of dynamic contact interaction, their solution is connected with severe mathematical and calculation difficulties. To overcome this impediment, a rich variety of approaches and methods have been suggested, and the overview of current results in the field can be found in recent state-of-the-art articles by Abrate [1], Rossikhin and Shitikova [2], and Qatu et al. [3].

In many engineering applications, it is important to understand the transient behaviour of isotropic as well as composite thin-walled shell structures subjected to central impact not only by a small projectile but by another shell as well. The problem on impact of a rigid body against an elastic spherical shell has been repeatedly considered by different authors using disparate models of shock interaction (the overview of the papers in the field could be found in [2] and [4]), elastic spherical shell impacted with an elastic barrier has been considered in [5], while the nonlinear collision of two shells, to the authors' knowledge, has not been yet analyzed analytically in the literature. The only paper considering the analysis of two colliding fractionally damped spherical shells in modelling blunt human head impacts has been recently appeared [6], wherein the contact force is represented using linear approach via fractional derivative standard linear solid model.

Recently Rossikhin and Shitikova [7] have developed a new formulation of the ray method which is applicable for analyzing the propagation of surfaces of strong and weak discontinuity in thin elastic bodies when the wave fronts and the rays are referenced to the curvilinear system of coordinates. It should be noted that the ray method is primarily used for obtaining the problem solution analytically. This approach is based on the reduction of the three-dimensional equations of the dynamic theory of elasticity, which first should be written in discontinuities, to the two-dimensional equations by virtue of integration over the coordinate perpendicular to the middle surface of a thin body. The recurrent equations of this ray method are free from the shear coefficient, which is usually inherent to the Timoshenko type theories, and involve only two elastic constants: Poisson's ratio and elastic modulus of elongation.

The theory proposed in [7] is applicable for short times after the passage of the wave front, but it possesses the simplicity inherent in the "classical" theory of thin bodies. The advantages of this approach have been readily illustrated by solving the engineering problems on normal impact of an elastic thin cylindrical and spherical projectiles against an elastic spherical shell, respectively, in [8] and [9]. Nonlinear Hertz's theory was employed within the contact region, resulting in the nonlinear differential equation with respect to the value characterizing the local indentation of the impactor into the target, the analytical solution of which was found in terms of time series with integer and fractional powers. It has been shown that the contact duration and the peak of the contact force gradually decrease for increasing shell curvature. The similar conclusion concerning the contact duration can be found in [10].

In the present paper, the analytical approach proposed in [4] for the analysis of the dynamic response of the elastic isotropic spherical shell subjected to the impact by elastic spherical and long cylindrical hemisphere-nose projectiles has been extended to the problem of the collision of two elastic spherical shells.

\section{PROBLEM FORMULATION}

In order to model the dynamic response of two colliding spherical shells, let us consider two spherical shells moving one after another along the line intersecting their centers of gravity with the velocities $V_{01}$ and $V_{02}$, in so doing the velocity of the second shell $V_{02}$ is larger than that of the first one $V_{01}$. The shells' radii $R_{1}$ and $R_{2}$, their densities $\varrho_{1}$ and $\varrho_{2}$, and elastic constants of the materials they are made of are different. The impact occurs at the moment $t=0$. At the moment of impact, at the point of tangency (or of contact) of the two spheres, two shock waves (surfaces of strong discontinuity) are generated which then propagate along spherical surfaces with the velocities of elastic waves. Behind the waves fronts, the solution could be constructed in terms of one-term ray expansions obtained via the theory of discontinuities. 


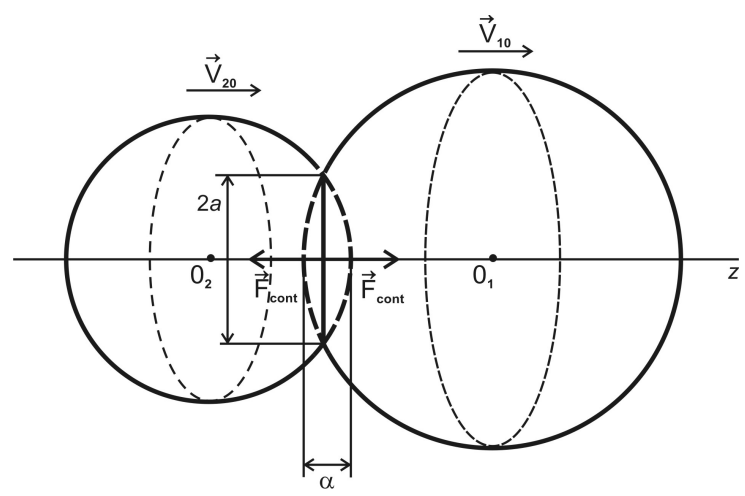

Fig. 1. Scheme of the Elastic Central Impact of Two Spherical Shells

The form of the solution within the contact domain, the dimension of which in the general case is a function of time, depends on the type of the material the colliding bodies are made of. Thus, in the case of the collision of two elastic spherical shells (Figure 1), the solution in the contact domain could be found using Hertz' theory.

\section{GOVERNING EQUATIONS}

Since at the moment of impact, two shock waves (surfaces of strong discontinuity) are generated at the point of tangency (or the point of contact) of the two colliding spheres, which then propagate along spherical surfaces with the velocities of elastic waves, then behind the waves fronts the solution could be constructed in terms of one-term ray expansions [7] obtained via the theory of discontinuities [2].

\section{A. The Main Kinematic and Dynamic Characteristics of the Wave Surface}

To construct the ray expansions, we shall use two conditions of compatibility for the values to be found [11]: the geometric condition of compatibility

$$
\left[u_{i, j}\right]=\left[\frac{\mathrm{d} u_{i}}{\mathrm{~d} n}\right] \lambda_{j}+\left[\frac{\mathrm{d}\left(u_{i} \xi_{j}\right)}{\mathrm{d} \xi}\right],
$$

and the kinematic condition of compatibility

$$
\left[v_{i}\right]=-\left[\frac{\mathrm{d} u_{i}}{\mathrm{~d} n}\right] G,
$$

in so doing the geometric condition of compatibility is written with due account for the fact that the wave surface represents itself a 'wave-strip' (Figure 2).

Here $u_{i}$ are the displacement vector components, $v_{i}=$ $\partial u_{i} / \partial t, G$ is the normal velocity of the wave surface, $x_{i}$ are the spatial rectangular coordinates, $t$ is the time, $\mathrm{d} / \mathrm{d} n$ is the derivative with respect to the normal to the wave surface, $\xi$ is the coordinate directed along the normal to the spherical shell, $\lambda_{i}$ and $\xi_{i}$ are the components of the unit vectors $\vec{\lambda}$ and $\vec{\xi}$ directed along the normal to the wave and spherical surfaces, respectively, $[Z]=Z^{+}-Z^{-}$is the discontinuity in the desired field $Z$, where "+" and "-" denote that the given value is calculated "ahead of" and "behind" the surface of strong discontinuity, and Latin indices take on the values 1,2,3.

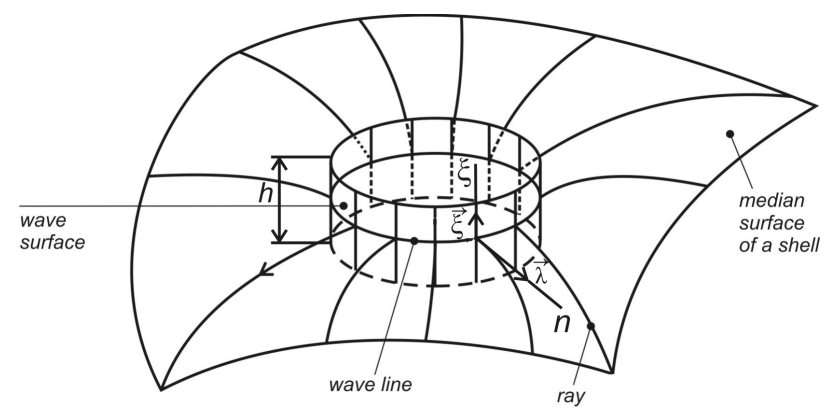

Fig. 2. Scheme of the Propagating Wave-strip along the Spherical Shell Surface

Eliminating the value $\left[\mathrm{d} u_{i} / \mathrm{d} n\right]$ from (1) and (2), we find

$$
\left[u_{i, j}\right]=-G^{-1}\left[v_{i}\right] \lambda_{j}+\left[\frac{\mathrm{d}\left(u_{i} \xi_{j}\right)}{\mathrm{d} \xi}\right] .
$$

Writing the Hooke's law for a three-dimensional medium in terms of discontinuities and using the condition of compatibility (3), we have

$$
\begin{aligned}
{\left[\sigma_{i j}\right] } & =-G^{-1} \lambda\left[v_{\lambda}\right] \delta_{i j}-G^{-1} \mu\left(\left[v_{i}\right] \lambda_{j}+\left[v_{j}\right] \lambda_{i}\right) \\
& +\lambda\left[u_{\xi, \xi}\right] \delta_{i j}+\mu\left(\left[\frac{\mathrm{d}\left(u_{i} \xi_{j}\right)}{\mathrm{d} \xi}+\frac{\mathrm{d}\left(u_{j} \xi_{i}\right)}{\mathrm{d} \xi}\right]\right),
\end{aligned}
$$

where $\left[\sigma_{i j}\right]$ are jumps of the stress tensor components, $\lambda$ and $\mu$ are Lamé constants, $\delta_{i j}$ is the Kronecker's symbol,

$$
\left[v_{\lambda}\right]=\left[v_{i}\right] \lambda_{i}, \quad\left[u_{\xi, \xi}\right]=\left[\frac{\mathrm{d}\left(u_{i} \xi_{i}\right)}{\mathrm{d} \xi}\right]=\left[\frac{\mathrm{d} u_{\xi}}{\mathrm{d} \xi}\right] .
$$

Multiplying relationship (4) from the right and from the left by $\xi_{i} \xi_{j}$ and considering equation

$$
\left[\sigma_{\xi \xi}\right]=\left[\sigma_{i j}\right] \xi_{i} \xi_{j}=0,
$$

what corresponds to the assumption that the normal stresses on the cross-sections parallel to the middle surface of the spherical shell could be neglected with respect to other stresses, we find

$$
\left[u_{\xi, \xi}\right]=\frac{\lambda}{G(\lambda+2 \mu)}\left[v_{\lambda}\right]
$$

Multiplying (4) from the right and from the left by $\lambda_{i} \lambda_{j}$, we are led to the equation

$$
\left[\sigma_{\lambda \lambda}\right]=\left[\sigma_{i j}\right] \lambda_{i} \lambda_{j}=-G^{-1}(\lambda+2 \mu)\left[v_{\lambda}\right]+\lambda\left[u_{\xi, \xi}\right] .
$$

Substituting (5) in (6) yields

$$
\left[\sigma_{\lambda \lambda}\right]=-\frac{4 \mu(\lambda+\mu)}{\lambda+2 \mu} G^{-1}\left[v_{\lambda}\right]
$$

or

$$
\left[\sigma_{\lambda \lambda}\right]=-\frac{E}{1-\sigma^{2}} G^{-1}\left[v_{\lambda}\right]
$$

where $\sigma$ is the Poisson's ratio.

Alternatively, multiplying the three-dimensional equation of motion written in terms of discontinuities

$$
\left[\sigma_{i j}\right] \lambda_{j}=-\rho G\left[v_{i}\right]
$$


by $\lambda_{i}$, we obtain

$$
\left[\sigma_{\lambda \lambda}\right]=-\rho G\left[v_{\lambda}\right]
$$

where $\rho$ is the density of the shell's material.

Eliminating the value $\left[\sigma_{\lambda \lambda}\right]$ from (7) and (9), we find the velocity of the quasi-longitudinal wave propagating in the spherical shell

$$
G_{1}=\sqrt{\frac{E}{\rho\left(1-\sigma^{2}\right)}}
$$

Relationship (7) with due account for (10) takes the form

$$
\left[\sigma_{\lambda \lambda}\right]=-\rho G_{1}\left[v_{\lambda}\right]
$$

Multiplying (4) by $\lambda_{i} \xi_{j}$ and (8) by $\xi_{i}$, we have

$$
\begin{aligned}
{\left[\sigma_{\lambda \xi}\right] } & =\left[\sigma_{i j}\right] \lambda_{i} \xi_{j}=-\mu G^{-1}\left[v_{\xi}\right] \\
{\left[\sigma_{\lambda \xi}\right] } & =-\rho G\left[v_{\xi}\right]
\end{aligned}
$$

where $\left[v_{\xi}\right]=\left[v_{i}\right] \xi_{i}$.

Eliminating the value $\left[\sigma_{\lambda \xi}\right]$ from (12) and (13), we find the velocity of the quasi-transverse wave

$$
G_{2}=\sqrt{\frac{\mu}{\rho}}
$$

Considering (14), relationship (12) takes the form

$$
\left[\sigma_{\lambda \xi}\right]=-\rho G_{2}\left[v_{\xi}\right]
$$

\section{B. Construction of One-term Ray Expansions}

Behind the front of each of two transient waves (surfaces of strong discontinuity) upto the boundary of the contact domain (Figure 1) relationships (11) and (15) are valid, which are the first terms of the ray expansions for the values $\sigma_{\lambda \lambda}, \sigma_{\lambda \xi}, v_{\lambda}$, and $v_{\xi}$ (Figure 3). Thus, within the entire disturbed domain it could be approximately considered that

$$
\begin{aligned}
\sigma_{\lambda \lambda}^{-} & =-\rho G_{1} v_{\lambda}^{-}, \\
\sigma_{\lambda \xi}^{-} & =-\rho G_{2} v_{\xi}^{-} .
\end{aligned}
$$

Knowing $v_{\lambda}^{-}$, and $v_{\xi}^{-}, \sigma_{\lambda \lambda}^{-}$, and $\sigma_{\lambda \xi}^{-}$, it is possible to calculate $v_{z}^{-}$, and $v_{r}^{-}$, and $\sigma_{r z}^{-}$according to the following formulas:

$$
\begin{aligned}
v_{z}^{-} & =v_{\xi}^{-} \sqrt{1-\frac{a^{2}}{R^{2}}}-v_{\lambda}^{-} \frac{a}{R} \\
v_{r}^{-} & =v_{\xi}^{-} \frac{a}{R}+v_{\lambda}^{-} \sqrt{1-\frac{a^{2}}{R^{2}}} \\
\sigma_{r z}^{-} & =-\sigma_{\lambda \lambda}^{-} \frac{a}{R} \sqrt{1-\frac{a^{2}}{R^{2}}}+\sigma_{\lambda \xi}^{-}\left(1-2 \frac{a^{2}}{R^{2}}\right)
\end{aligned}
$$

where $a$ is the radius of the contact spot.

Considering the cone angles of the contact spot $2 \gamma_{1}$ and $2 \gamma_{2}$ as small values, and putting $\cos \gamma_{\alpha} \approx 1, \sin \gamma_{\alpha} \approx \gamma_{\alpha}=a R_{\alpha}^{-1}$,

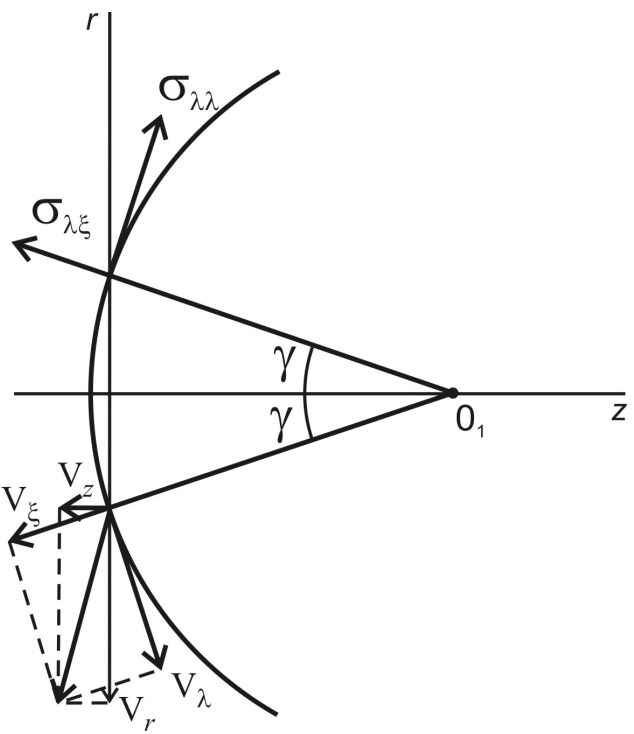

Fig. 3. Scheme of Velocities and Stresses in the Shell's Element on the Boundary of the Contact Domain

we obtain from (18)-(20)

$$
\begin{aligned}
\widetilde{v_{z}} & =\widetilde{v_{\xi}}-\widetilde{v_{\lambda}} \frac{a}{R}, \\
\widetilde{v_{r}} & =\widetilde{v_{\xi}} \frac{a}{R}+\widetilde{v_{\lambda}}, \\
\widetilde{\sigma_{r z}} & =\rho\left(G_{1} \widetilde{v_{\lambda}} \frac{a}{R}-G_{2} \widetilde{v_{\xi}}\right),
\end{aligned}
$$

where a tilde over a value labels that the corresponding value is calculated on the boundary of the contact domain, i.e. at $r=a$, and $r, \theta, z=x_{3}$ is the cylindrical set of coordinates with a center at the initial point of contact of two spherical shells (Figure 3).

\section{Central Impact of Two Elastic Spherical SHELLS}

In the case of the collision of two elastic spherical shells (Figure 1) the solution in the contact domain could be found using Hertz' theory. Thus, according the Hertzian law the contact force is defined as

$$
F_{\text {cont }}=k \alpha^{3 / 2},
$$

where $\alpha$ is the local bearing of the shells' materials, the indices 1 and 2 refer to the first and second shells, respectively,

$$
k=\frac{4}{3} \frac{\sqrt{R^{\prime}}}{k_{1}+k_{2}}, \quad \frac{1}{R^{\prime}}=\frac{1}{R_{1}}+\frac{1}{R_{2}}, \quad k_{i}=\frac{1-\sigma_{i}^{2}}{E_{i}}(i=1,2)
$$

Then the equations of motion of the contact domain of two spherical shells could be written in the form

$$
\begin{aligned}
& \rho_{1} \pi a^{2} h_{1} \widetilde{\dot{v}_{z 1}}=2 \pi a h_{1} \widetilde{\sigma_{r z 1}}+k \alpha^{3 / 2}, \\
& \rho_{2} \pi a^{2} h_{2} \widetilde{\dot{v}_{z 2}}=2 \pi a h_{2} \widetilde{\sigma_{r z 2}}-k \alpha^{3 / 2}
\end{aligned}
$$

where an overdot denotes the time-derivative. 
In order to calculate the values $\widetilde{\sigma_{r z \alpha}}$, let us use formulas (21)-(23) and consider that $\widetilde{v_{r}}=\dot{a}$. As a result we obtain

$$
\begin{aligned}
\widetilde{\sigma_{r z \alpha}} & =\rho_{\alpha}\left(G_{1}^{(\alpha)}-G_{2}^{(\alpha)}\right) \frac{\left(a^{2}\right)}{2 R_{\alpha}} \\
& -\rho_{\alpha}\left(G_{1}^{(\alpha)} \frac{a^{2}}{R_{\alpha}^{2}}+G_{2}^{(\alpha)}\right) \widetilde{v_{z \alpha}} .
\end{aligned}
$$

Substituting (27) in (25) and (26) and considering that $\dot{a}=R^{\prime} \alpha$, we find

$$
\begin{aligned}
\rho_{1} \pi R^{\prime} \alpha h_{1} \widetilde{\dot{v}_{z 1}} & =2 \pi R^{\prime 1 / 2} \alpha^{1 / 2} h_{1} \rho_{1}\left[\left(G_{1}^{(1)}-G_{2}^{(1)}\right) \frac{R^{\prime}}{2 R_{1}} \dot{\alpha}\right. \\
& \left.-\left(G_{1}^{(1)} \frac{R^{\prime}}{R_{1}^{2}} \alpha+G_{2}^{(1)}\right) \widetilde{v_{z 1}}\right]+k \alpha^{3 / 2}, \quad(28) \\
\rho_{2} \pi R^{\prime} \alpha h_{2} \widetilde{\dot{v}_{z 2}} & =2 \pi R^{\prime 1 / 2} \alpha^{1 / 2} h_{2} \rho_{2}\left[\left(G_{1}^{(2)}-G_{2}^{(2)}\right) \frac{R^{\prime}}{2 R_{2}} \dot{\alpha}\right. \\
& \left.-\left(G_{1}^{(2)} \frac{R^{\prime}}{R_{2}^{2}} \alpha+G_{2}^{(2)}\right) \widetilde{v_{z 2}}\right]-k \alpha^{3 / 2} . \quad(29)
\end{aligned}
$$

The following equation

$$
\widetilde{v_{z 2}}-\widetilde{v_{z 1}}=\dot{\alpha}-V
$$

should be added to equations (28) and (29), where

$$
V=V_{02}-V_{01} \text {. }
$$

The closed system of three equations (28)-(30) allows one to determine the desired values: $\widetilde{v_{z 1}}, \widetilde{v_{z 2}}$, and $\dot{\alpha}$.

It should be noted that the set of three equations (28)-(30) could be reduced to a set of two equations after the substitution of the independent variable, i.e., the substitution of $t$ by $\alpha$. In this case, considering (30) it could be written

$$
\begin{aligned}
& \widetilde{v_{z 1}}=\frac{\widetilde{v_{z 1}}}{d \alpha} \dot{\alpha}=\frac{\widetilde{v_{z 1}}}{d \alpha}\left[\left(\widetilde{v_{z 2}}-\widetilde{v_{z 1}}\right)+V\right], \\
& \widetilde{v_{z 2}}=\frac{\widetilde{v_{z 2}}}{d \alpha} \dot{\alpha}=\frac{\widetilde{v_{z 2}}}{d \alpha}\left[\left(\widetilde{v_{z 2}}-\widetilde{v_{z 1}}\right)+V\right] .
\end{aligned}
$$

Then, in view of (31) and (32), equations (28) and (29) take the form

$$
\begin{aligned}
& \alpha^{1 / 2} \frac{\widetilde{v_{z 1}}}{d \alpha}\left(\widetilde{v_{z 2}}-\widetilde{v_{z 1}}+V\right) \\
& =2 R^{\prime-1 / 2}\left[\left(G_{1}^{(1)}-G_{2}^{(1)}\right) \frac{R^{\prime}}{2 R_{1}}\left(\widetilde{v_{z 2}}-\widetilde{v_{z 1}}+V\right)\right. \\
& \left.-\left(G_{1}^{(1)} \frac{R^{\prime}}{R_{1}^{2}} \alpha+G_{2}^{(1)}\right) \widetilde{v_{z 1}}\right]+\frac{k \alpha}{\pi h_{1} \rho_{1} R^{\prime}}, \\
& \alpha^{1 / 2} \frac{\widetilde{v_{z 2}}}{d \alpha}\left(\widetilde{v_{z 2}}-\widetilde{v_{z 1}}+V\right) \\
& =2 R^{\prime-1 / 2}\left[\left(G_{1}^{(1)}-G_{2}^{(1)}\right) \frac{R^{\prime}}{2 R_{2}}\left(\widetilde{v_{z 2}}-\widetilde{v_{z 1}}+V\right)\right. \\
& \left.-\left(G_{1}^{(1)} \frac{R^{\prime}}{R_{1}^{2}} \alpha+G_{2}^{(1)}\right) \widetilde{v_{z 2}}\right]+\frac{k \alpha}{\pi h_{1} \rho_{1} R^{\prime}} .
\end{aligned}
$$

Equations (33) and (34) are more suitable for numerical analysis than equations (28) and (29), and allow one to find $\widetilde{v_{z 1}}$ and $\widetilde{v_{z 1}}$ as functions of $\alpha$.
A. Solution in the Case of the Collision of Two Similar Spherical Shells

Suppose that the spherical shells are made of the same material and have equal dimensions, i.e. $\rho=\rho_{1}=\rho_{2}, h=$ $h_{1}=h_{2}, G_{1}=G_{1}^{(1)}=G_{1}^{(2)}, G_{2}=G_{2}^{(1)}=G_{2}^{(2)}$, and $R^{\prime}=$ $1 / 2 R$. Then subtracting (28) from (29) with due account for (30), we have

$$
\begin{aligned}
\alpha \ddot{\alpha} & +\frac{2 \sqrt{2}}{\sqrt{R}}\left(G_{1} \frac{1}{2 R} \alpha+G_{2}\right) \alpha^{1 / 2} \dot{\alpha}+\frac{4 k}{\rho \pi h R} \alpha^{3 / 2} \\
& =\frac{2 \sqrt{2}}{\sqrt{R}}\left(G_{1} \frac{1}{2 R} \alpha+G_{2}\right) \alpha^{1 / 2} V
\end{aligned}
$$

We will seek a solution of (35) in the form of the following series with respect to time $t$ :

$$
\alpha=V t+\sum_{i=1}^{\infty} c_{i} t^{(2 i+1) / 2}+\sum_{j=2}^{\infty} d_{j} t^{j},
$$

where $c_{i}$ and $d_{j}$ are coefficients to be determined.

Substituting (36) into Eq. (35) and equating the coefficients at integer and fractional powers of $t$, we are led to the set of equations for defining the coefficients $c_{i}$ and $d_{j}$. For example, the first seven of them have the form

$$
\begin{gathered}
c_{1}=d_{2}=c_{3}=0, \quad c_{2}=-\frac{16}{15} \frac{k}{\rho \pi h R} V^{1 / 2}<0 \\
d_{3}=\frac{8 \sqrt{2}}{9} \frac{k G_{2}}{\rho \pi h R^{3 / 2}}>0 \\
d_{4}=\frac{4}{45} \frac{k V}{\rho \pi h R^{2}}\left(\frac{5 \sqrt{2} G_{1}}{\sqrt{R}}+\frac{6 k}{\rho \pi h V}\right)>0 \\
c_{4}=\frac{4 \sqrt{2}}{63} \frac{c_{2} G_{2}}{R^{3 / 2} V}\left[\frac{3 k}{\rho \pi h}+\frac{5 \sqrt{2}}{3} \frac{V G_{1}}{\sqrt{R}}\right]<0
\end{gathered}
$$

and thus the function $\alpha$ is written as

$$
\alpha=V t+c_{2} t^{5 / 2}+d_{3} t^{3}+d_{4} t^{4}+c_{4} t^{9 / 2} .
$$

Substitution of (37) in (24) allows one to determine the contact force in this case.

If we limit ourselves by the first two terms in (37), i.e., utilize only the quasi-static approximation, then we can obtain the first approximations for the contact time $t_{\text {cont }}$ and the time $t_{\max }$ when the contact force attains its maximal magnitude $F_{\max }=k \alpha_{\max }^{3 / 2}$ :

$$
\begin{aligned}
t_{\text {cont }} & =\left(-\frac{V}{c_{2}}\right)^{2 / 3} \\
& =\left(\frac{15}{16} \frac{\rho \pi h R V^{1 / 2}}{k}\right)^{2 / 3} \\
t_{\max } & =\left(\frac{3}{18} \frac{\rho \pi h R V^{1 / 2}}{k}\right)^{2 / 3} \\
F_{\max } & =\frac{9 \sqrt{3}}{40 \sqrt{5}} \rho \pi h R V^{2} \\
& \approx 0.174 \rho \pi h R V^{2} .
\end{aligned}
$$


Reference to Eqs. (38)-(40) shows that the maximum of the contact force and the duration of contact depend only on the difference of velocities $V=V_{02}-V_{01}$ and are independent of the velocities of the transient waves propagating in the colliding bodies. To take them into account, it is necessary to use the series (36) for the value $\alpha$ involving at least its five or more terms.

\section{B. Solution in the Case of Different Shells without Regard for the Inertia of the Contact Domain}

If the inertia of the contact spot is neglected in Eqs. (28) and (29), then they could be rewritten in the form

$$
\begin{aligned}
& a_{1} \dot{\alpha}-\widetilde{v_{z 1}}+b_{1} \alpha=0, \\
& a_{2} \dot{\alpha}-\widetilde{v_{z 2}}-b_{2} \alpha=0,
\end{aligned}
$$

where

$$
a_{\alpha}=\frac{\left(G_{1}^{(\alpha)}-G_{2}^{(\alpha)}\right) R^{\prime}\left(2 R_{\alpha}\right)^{-1}}{G_{1}^{(\alpha)} R^{\prime} R_{\alpha}^{-2} \alpha+G_{2}^{(\alpha)}}
$$

and

$$
b_{\alpha}=\frac{k}{2 \pi \sqrt{R^{\prime}} h_{\alpha} \rho_{\alpha}\left(G_{1}^{(\alpha)} R^{\prime} R_{\alpha}^{-2} \alpha+G_{2}^{(\alpha)}\right)} .
$$

Subtracting (41) from (42) with due account for (30) yields

$$
\dot{\alpha}+\frac{b_{1}+b_{2}}{1-\left(a_{2}-a_{1}\right)} \alpha=\frac{V}{1-\left(a_{2}-a_{1}\right)} .
$$

Since $\alpha$ is a small value, then it is possible to neglect the value $G_{1} R^{\prime} R_{\alpha}^{-2} \alpha$ as compared with the magnitude of $G_{2}$; the values $a_{1}$ and $a_{2}$ are smaller than unit, that is why the value $a_{2}-a_{1}$ could be ignored with respect to the unit. Then Eq. (43) takes the form

$$
\dot{\alpha}+\left(b_{1}+b_{2}\right) \alpha=V
$$

where $b_{1}$ and $b_{2}$ are constants.

Integrating Eq. (44) and considering the initial conditions

$$
\left.\alpha\right|_{t=0}=0,\left.\quad \dot{\alpha}\right|_{t=0}=V,
$$

we find

$$
\alpha=\frac{V}{b_{1}+b_{2}}\left(1-\mathrm{e}^{-\left(b_{1}+b_{2}\right) t}\right)
$$

Note that the law of variation in the local bearing (the law of the force changes) defined by (46) qualitatively coincides with the form of AGB curve presented in Figure 45 in [12], wherein the characteristic law of the force changes during the contact of two spherically-headed rods is shown.

The local bearing of two colliding spherical shells increases until the moment when reflected waves (waves of 'unloading') return to the contact zone. Each of the reflected waves unloads the contact domain, resulting in the rebound of contacting shells.

\section{Conclusion}

In the present paper, the collision of two elastic spherical shells is investigated considering that the impact process is accompanied by the generation and propagation of quasilongitudinal and quasi-transverse shock waves, amplitudes of which are proportional to $\sin ^{-1 / 2} \varphi$ [7]. If the contact zone is located at $\varphi=0$, then at $\varphi=\pi$, i.e., on the other end of the diameter, there could occur the focusing of these waves.

The solution behind the wave fronts has been constructed with the help of the theory of discontinuities under the assumption that the reflected waves approach the contact zone after the termination of the impact process. Since the local bearing of the materials of the colliding elastic shells has been taken into account, then the solution in the contact domain has been found via the Hertz contact theory.

\section{ACKNOWLEDGMENT}

The research described in this publication was made possible by Grant No. 2012-14.B37.21.0379-1-001 from the Ministry of Education and Science of the Russian Federation.

\section{REFERENCES}

[1] S. Abrate, Modeling of impacts on composite structures, Composite Structures, vol. 51, pp. 129-138, 2001.

[2] Yu. A. Rossikhin and M. V. Shitikova, Transient response of thin bodies subjected to impact: Wave approach, Shock and Vibration Digest, vol. 39, pp. 273-309, 2007.

[3] M. S. Qatu, R. W. Sullivan and W. Wang, Recent research advances on the dynamic analysis of composite shells: 2000-2009, Composite Structures, vol. 93, pp. 14-31, 2010.

[4] Yu. Rossikhin, M. Shitikova, and V. Shamarin, Dynamic response of spherical shells impacted by falling objects, International Journal of Mechanics, vol. 3, pp. 166-182, 2011.

[5] S. M. Baghaei, A. M. Sadegh, Elastic spherical shell impacted with an elastic barrier: A closed form solution, International Journal of Solids and Structures, vol. 48, 3257-3266, 2011.

[6] Yu. A. Rossikhin and M. V. Shitikova, Analysis of two colliding fractionally damped spherical shells in modelling blunt human head impacts, Central European Journal of Physics, vol. 11, 2013, DOI: $10.2478 / \mathrm{s} 11534-013-0194-4$.

[7] Yu. A. Rossikhin and M. V. Shitikova, The method of ray expansions for investigating transient wave processes in thin elastic plates and shells, Acta Mechanica, vol. 189, pp. 87-121, 2007.

[8] Yu. Rossikhin and M. Shitikova, "Dynamic response of a spherical shell impacted by an elastic rod with a rounded end," in Latest Trends on Engineering Mechanics, Structures, Engineering Geology, Proc. of the 3rd WSEAS Int. Conf. EMESEG'10, WSEAS Press, ISBN 978-960474-203-5, 2010, pp. 453-458.

[9] Yu. Rossikhin, M. Shitikova, and V. Shamarin, "Dynamic response of a spherical shell impacted by a sphere," in Recent Research in Hydrology, Geology and Continuum Mechanics, Proc. of the 6th IASME/WSEAS Int. Conf. on Continuum Mechanics (CM'11), WSEAS Press, ISBN 978-960-474-275-2, 2011, pp. 51-56.

[10] M. G. Koller and M. Busenhart, Elastic impact of spheres on thin shallow spherical shells, International Journal of Impact Engineering, vol. 4, pp. 11-21, 1986.

[11] T. Y. Thomas, Plastic Flow and Fracture in Solids. New York: Academic Press, 1961.

[12] W. Goldsmith, Impact. London: Arnold, 1960.

\section{Creative Commons Attribution License 4.0} (Attribution 4.0 International, CC BY 4.0)

This article is published under the terms of the Creative Commons Attribution License 4.0

https://creativecommons.org/licenses/by/4.0/deed.en US 